\title{
Dust in the Tycho, Kepler and Crab supernova remnants ${ }^{\star}$
}

\author{
T. Douvion ${ }^{1,2}$, P. O. Lagage ${ }^{1}$, C. J. Cesarsky ${ }^{3}$, and E. Dwek ${ }^{4}$ \\ 1 DSM/DAPNIA/Service d'Astrophysique, CEA/Saclay, 91191 Gif-sur-Yvette, France \\ 2 Supélec, Service des mesures, 91192 Gif-sur-Yvette, France \\ 3 European Southern Observatory, Karl Schwarzchild Strasse 2, 85748 Garching bei München, Germany \\ 4 NASA GSFC, Code 685, Greenbelt, MD 20771, USA
}

Received 14 December 2000 / Accepted 9 February 2001

\begin{abstract}
SuperNova Remnants (SNR) have been extensively studied in radio, X-rays and optical, but barely in the InfraRed (IR). From IRAS observations, SNR are known to be IR emitters, but the origin of the emission is difficult to assess because of the limited angular and spectral resolution of IRAS observations. ISO follow-up observations of the Cassiopeia-A SNR have shown that the mid-IR radiation from this remnant was dominated by thermal emission from silicate condensates made from SN material. These condensates constitute a probe which can provide unique information about element mixing inside a SN. In this paper, we present ISOCAM observations of the three other young SNR's known in our galaxy: the Kepler SNR, the Tycho SNR and the Crab Nebula. The emission observed from the Kepler SNR is dominated by dust thermal emission. The dust at the origin of the IR emission is circumstellar, not from SN condensates. "Astronomical" silicates, collisionally heated to a temperature of $107 \mathrm{~K}$, can account for both the mid-IR ISO data and the IRAS data. The IR emission from Tycho is probably also emitted by circumstellar or interstellar dust. In order to reproduce both the mid-IR ISO data and the IRAS data, a model with two dust components, one at a temperature of $107 \mathrm{~K}$ and the other at $55 \mathrm{~K}$, is needed. The mid-IR emission from the Crab is dominated by synchrotron radiation; no dust is detected.
\end{abstract}

Key words. ISM: supernova remnants - individual object: Kepler, Crab, Tycho

\section{Introduction}

SuperNovae (SNe) are key objects in the Universe (e.g. review by Trimble 1983 and references therein). They are the factories which feed the interstellar medium with many of the heavy elements. The idea that part of the SN ejecta condense to form dust was introduced more than 30 years ago (Cernushi et al. 1967; Hoyle \& Wickramasinghe 1970). Laboratory studies of meteorites provided the first indications that part of the interstellar dust does originate from $\mathrm{SNe}$. Indeed, $\mathrm{SNe}$ are the only place where nucleosynthesis can generate the isotopic anomalies observed in meteorites (e.g. Zinner 1996 and references therein). The observations of SN1987A have indirectly indicated that dust was formed about one year after the SN explosion (Lucy et al. 1989; Moseley et al. 1989; Bouchet et al. 1991; Suntzeff et al. 1991; Wooden et al. 1993; Colgan et al. 1994). Dust formation models have been developed to account

Send offprint requests to: T. Douvion or P. O. Lagage, e-mail: tdouvion@cea.fr; lagage@cea.fr

* Based on observations with ISO, an ESA project with instruments funded by ESA Member States (especially the PI countries: France, Germany, The Netherlands and the UK) and with the participation of ISAS and NASA, and on observations obtained at the ESO LaSilla Observatory, Prg 59.A-9905. for these observations (Kozasa et al. 1991; Kozasa et al. 1997). Another way to search for SN dust is to observe the InfraRed (IR) emission from SuperNova Remnants (SNR). Indeed, models indicate that dust, collisionally heated by the hot electrons of the plasma filling the SNR, can reach a temperature high enough so that the dust thermal emission peaks in the IR (Ostriker \& Silk 1973; Silk \& Burke 1974; Draine \& Salpeter 1979; Dwek 1986). Observations with the IRAS satellite have shown that IR radiation is emitted from many SNR (Arendt 1989; Saken et al. 1992). But, given the limited angular and spectral resolution of IRAS, it is difficult to disentangle the various contributions to the IR emission, such as ionic lines, synchrotron radiation, thermal emission from interstellar/circumstellar dust or from SN dust. The Infrared Space Observatory (ISO) (Kessler et al. 1996), thanks to a good spatial and spectral resolution, was well adapted to IRAS follow-up observations of SNR. ISO observations of the Cassiopeia A SNR (Cas A) have revealed the presence in this remnant of dust made of SN material (Lagage et al. 1996; Arendt et al. 1999). Studies of SN dust is not only interesting to assess the relative importance of $\mathrm{SNe}$ as interstellar dust manufacturers, but it can also provide unique information about the element mixing during the 
SN explosion, as shown in the case of Cas A (Douvion et al. 1999). In this paper, we present ISO observations of 3 other young galactic SNR: the Kepler SNR, the Tycho SNR and the Crab Nebula. Section 2 deals with the Kepler SNR. Section 3 is devoted to the Tycho SNR. The observations of the Crab Nebula are discussed in Sect. 4. A general discussion of the results is given in Sect. 5 .

\section{Kepler}

The Kepler SNR is the remnant of a SN explosion which was observed by Johannes Kepler in 1604. The size of the remnant on the sky is about $3^{\prime}$, which corresponds to a physical diameter of $3 \mathrm{pc}$ at the distance of the remnant, estimated to be $3.4 \mathrm{kpc}$ (Schaefer 1996 and references therein). The remnant has been extensively studied in X-rays (Hughes 1999; Rothenflug et al. 1994; Decourchelle \& Ballet 1994), radio (Dickel et al. 1988), optical (Blair et al. 1991), but barely in the IR. The only results come from IRAS observations (Dwek et al. 1987; Braun 1987; Arendt 1989; Saken et al. 1992).

\subsection{ISOCAM observations}

The Kepler SNR was extensively observed with ISOCAM, the camera on board of ISO (Cesarsky et al. 1996). The first observation was performed on 16 February, 1996; it consists of a full mapping of the SNR at a spatial resolution of $6^{\prime \prime}$ with the LW8 filter. The LW8 filter was chosen because its wavelength range, centered at $11.3 \mu \mathrm{m}$ and with a Full Width Half Maximum $(F W H M)$ of $1.3 \mu \mathrm{m}$, probes mainly continuum emission; only the [SIV] forbidden ionic lines at $10.5 \mu \mathrm{m}$, if present, may weakly contribute to the flux. With the 32 by 32 pixel detector array, a $3^{\prime}$ by $3^{\prime}$ field of view was covered by one frame. A raster map ( 3 by 2 frames along the $x$ and $y$ axis of the satellite) has been obtained to map the whole remnant, to have access to the adjacent sky background and to detect stars to improve the astrometry (see Sect. 2.3). An overlap of $36^{\prime \prime}$ between two subsequent frames was set, so that a total field of $8.4^{\prime} \times 5^{\prime}$ was covered by the observations. An integration time of $212 \mathrm{~s}$ (21 images with an elementary integration time of $10.1 \mathrm{~s}$ ) was spent on each raster position.

Data reduction was performed with the Cam Interactive Analysis ${ }^{1}$ package in five main steps. The first consists of removing from the signal the contribution of the detector dark current. The dark current behavior is well known and the dark current subtraction induces negligible errors. The second step consists of removing the glitches generated by cosmic-ray impacts; we used the powerful multi-resolution algorithm developed by Stark et al. (1995) and checked the results by visual inspection of the images. The signal is then corrected for detector memory effects; the recent algorithm based on the

\footnotetext{
${ }^{1}$ CIA is a joint development by the ESA astrophysics division and the ISOCAM PI, C. J. Cesarsky, Direction des sciences de la matière, CEA, France.
}

Foucks-Schubert modelisation of Si-Ga detectors (Coulais \& Abergel 1999) was used. The fourth step consists of correcting the data for the variation of the detector pixel responses (flat fielding). The flat-field image used for the correction was the median image of the 6 frames of the raster. Finally, the sky background (essentially due to the zodiacal light) was removed using the frames of the raster without emission from stars or from the remnant. The data then were calibrated in flux using the ISOCAM calibration database. The final result, limited to the $3^{\prime}$ by $3^{\prime}$ field where emission from the remnant is detected, is shown in Fig. 1.

In order to check the assumption that the emission in the LW8 is thermal dust emission, and in order to determine the nature of this dust (silicate, carboneous) and its temperature, follow-up observations, taking advantage of the spectro-imaging capabilities of ISOCAM, were performed on 1 March, 1998. In the spectro-imaging mode, a cube of images at different wavelengths is obtained by rotating a Circular Variable Filter (CVF), whose spectral resolution $(R=\lambda / \delta \lambda)$ is about 40 . The $16 \mu \mathrm{m}$ to $6.5 \mu \mathrm{m}$ wavelength range was scanned with 98 different CVF positions, starting with the longest wavelength. The CVF scan was first performed on a frame centered on the remnant and then repeated on a nearby field (RA $17^{\mathrm{h}} 30^{\mathrm{m}} 40.5$, Decl. $\left.-21^{\circ} 25^{\prime} 20^{\prime \prime} 2000\right)$ to record the sky background. A total integration time of $23 \mathrm{~s}$ (11 exposures of $2.1 \mathrm{~s}$ each) was spent for each CVF position. The data were reduced using the same method as for the LW8 filter image, except that we used a flat-field correction image from the ISOCAM database. Five typical spectra are shown in Fig. 2. The overall spectrum, obtained by co-adding all the pixels in regions $a$ to $e$ of Fig. 2, is displayed in Fig. 3.

All the spectra feature a strong continuum emission. At the brightest positions (spectra $a$ and $b$ on Fig. 2), we can recognize two ionic lines, the [ArII] line at about $7.0 \mu \mathrm{m}$, and the [NeII] line at $12.8 \mu \mathrm{m}$. The error bars correspond to a conservative uncertainty of $\pm 20 \%$, or to a $\pm 2 \mathrm{mJy}$ per pixel level when the flux is lower than $10 \mathrm{mJy}$. This is much higher than for the LW8 filter, but CVF filters are subject to relatively strong internal ghosts that make the calibration more difficult to perform.

\subsection{Photometry}

In the LW8 image, the total flux integrated over a circle with a 1.8 arcmin radius centered on the center of the remnant derived from X-ray observations (RA $17^{\mathrm{h}} 30^{\mathrm{m}} 41 \mathrm{~s} .5$, Decl. $-21^{\circ} 29^{\prime} 23^{\prime \prime}$ (2000), Smith et al. 1989) is found to be $1.0 \mathrm{Jy}$, with an uncertainty of $\pm 5 \%$. When convolving the global ISOCAM spectrum of Fig. 3 with the transmission of the LW8 filter, a flux of only $0.77 \mathrm{Jy}$ is found with an uncertainty of $\pm 20 \%$; the two results are compatible given the uncertainties. When convolving the spectrum with the transmission of the IRAS band 1 filter, a flux value of $1 \mathrm{Jy}$ is found. This value is on the low side of the IRAS value given by Saken et al. (1992) (1.8 $\pm 0.9 \mathrm{Jy})$, but is in good 


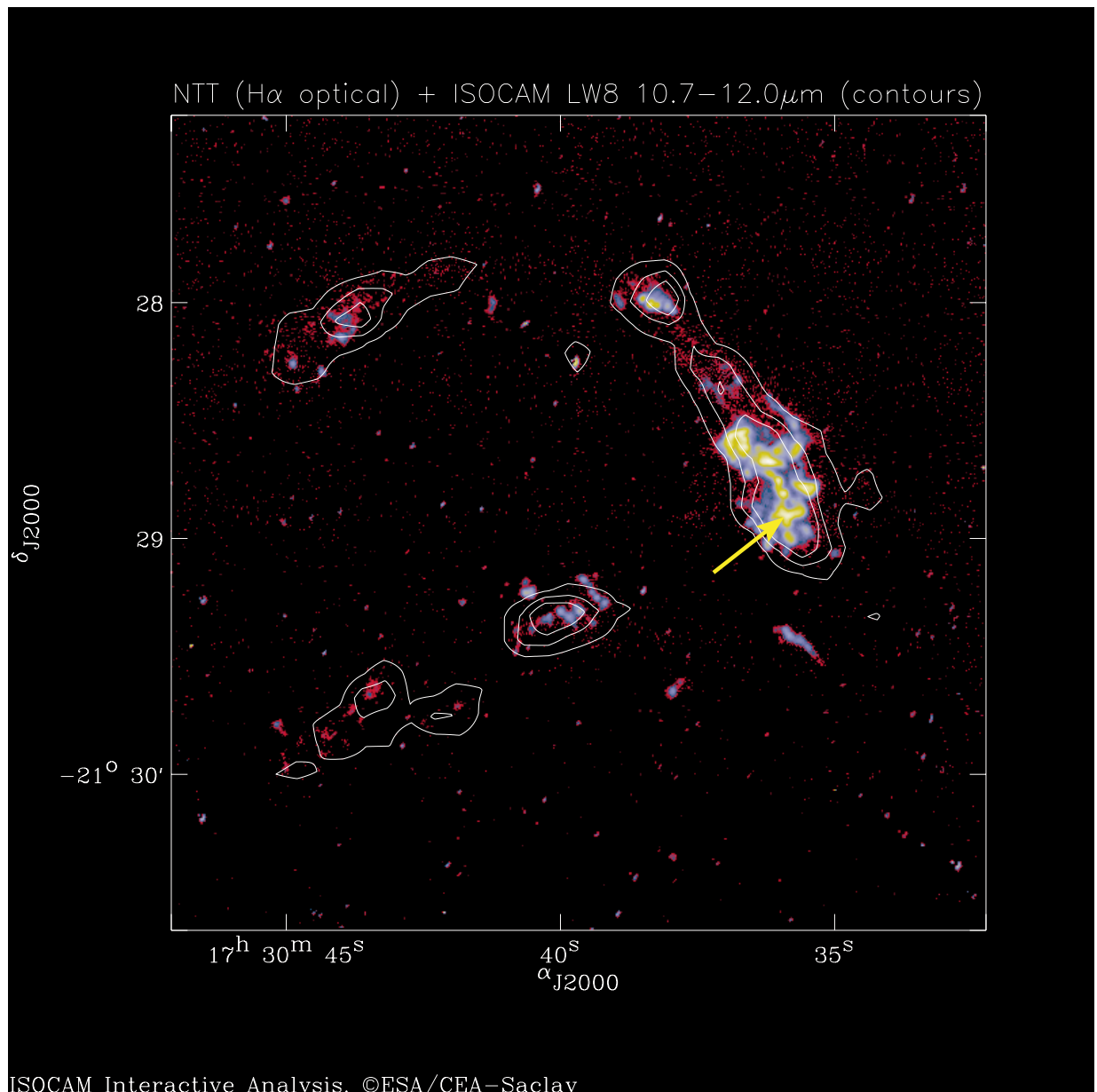

Fig. 1. Contours of the ISOCAM image of the Kepler SNR as observed with the LW8 filter (10.7$12 \mu \mathrm{m})$. The highest contour represents a flux level of $7.6 \mathrm{mJy}$ per ISOCAM pixel $\left(6^{\prime \prime} \times 6^{\prime \prime}\right)$, obtained at the position of the yellow arrow; the contour level is reduced by $2.5 \mathrm{mJy}$ at each subsequent contour. Underlying the IR contour map is an $\mathrm{H}_{\alpha}$ image of Kepler (with star contribution removed). The background $\mathrm{H}_{\alpha}$ image was taken from the archive of the NTT (New Technology Telescope) of the ESO La-Silla observatory. This is a $600 \mathrm{~s}$ exposure with a pixel field of view of $0.35^{\prime \prime}$ by $0.35^{\prime \prime}$. Color levels in the image go linearly from black-red for low values to blue-green-white for high values.

agreement with the mean value given by Arendt (1989) $(1.0 \pm 0.5 \mathrm{Jy}$ at $12 \mu \mathrm{m})$. In the following, we will adopt the Arendt values for the IRAS fluxes at 25, 60 and $100 \mu \mathrm{m}$ (respectively $10.1 \pm 1.0 \mathrm{Jy}, 7.1 \pm 0.6 \mathrm{Jy}, 2.9 \pm 1.1 \mathrm{Jy}$ ).

\subsection{Astrometry}

In order to compare the mid-IR image with other images (especially optical images), an astrometry precision at the arcsec level is required. The pointing uncertainties of the ISO satellite is in the $5^{\prime \prime}$ range. It is possible to improve the astrometry in the case of the Kepler LW8 image, because several stars are detected within the field of the LW8 raster map. There are too many stars in the Digital Sky Survey (DSS) to identify straightforwardly the stars seen by ISO. Images in the near-IR are needed to identify the reddest stars of the DSS, which are the mid-IR emitters. The observations were performed on 23 March, 1999 with the SOFI instrument mounted on the NTT (New Technology Telescope) of the European Southern Observatory (ESO). These observations were done in service mode. Three standard filters were used: the $J$ filter $(\lambda=1.25 \mu \mathrm{m}, F W H M=0.29 \mu \mathrm{m})$, the $H$ filter $(\lambda=1.65 \mu \mathrm{m}, F W H M=0.30 \mu \mathrm{m})$, and the $K \mathrm{~s}$ filter $(\lambda=2.16 \mu \mathrm{m}, F W H M=0.27 \mu \mathrm{m})$. The total integration time was $360 \mathrm{~s}$ in $J, 240 \mathrm{~s}$ in $H$ and $300 \mathrm{~s}$ in $K \mathrm{~s}$.
Several hundred stars were found, and it was possible to identify the reddest stars of the DDS. A $6^{\prime \prime}$ shift of the ISOCAM image was needed to correlate spatially those reddest stars with the stars detected in the mid-IR. The remaining astrometrical uncertainty is of $2^{\prime \prime}$ in both RA and Decl. directions. Note that an emission from the remnant itself was detected in regions $a$ and $b$ of Fig. 2 , in the $J$ and $H$ bands, but not in the $K$ band. It is difficult to give a precise estimate of the flux emitted by the remnant, because it is contaminated by the numerous field stars.

\subsection{Dust origin and composition}

The spectral information obtained from ISOCAM observations allows us to derive information on the origin of the IR emission. The global spectrum shown in Fig. 3 is well fitted by dust thermal emission; in the fit, the dust composition was that of "astronomical silicate", as defined by Draine \& Lee (1984); a blackbody or a modified blackbody (i.e. grains with an emissivity varying as $\lambda^{-1}$ or $\lambda^{-2}$, $\lambda$ being the wavelength) cannot fit the spectrum. Another advantage of using "astronomical silicates" is that both the mid-IR ISOCAM data and the IRAS data at 12, 25, 60 and $100 \mu \mathrm{m}$ can be fitted with a single grain temperature of $107 \mathrm{~K}$; previous models of the IRAS data required two dust components, a hot dust at $140 \mathrm{~K}$ and a cold dust 

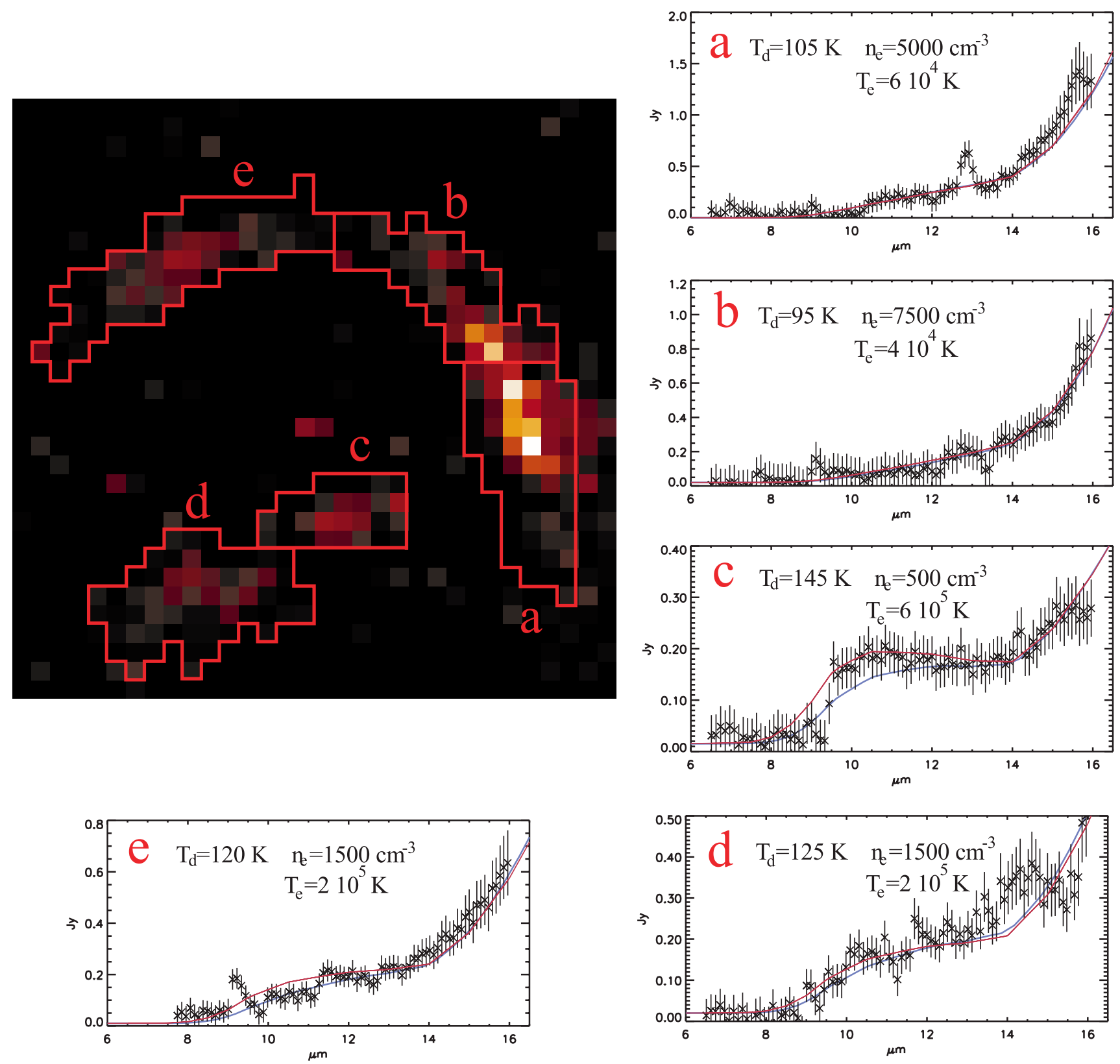

Fig. 2. ISOCAM image of the Kepler SNR where 5 regions, labelled $a$ to $e$, are delimited. The spectroscopic information on these regions, as obtained with ISOCAM spectro-imaging CVF observations, are shown in plots $a$ to $e$; (spectral information is available for each ISOCAM pixel, but the spatial resolution has been degraded to the 5 regions $a$ to $e$, in order to gain in terms of signal to noise; these regions account for $92 \%$ of the total flux, (as derived from the LW8 image)). The ISOCAM image shown here was obtained by stacking the 21 images corresponding to spectro-imaging observations between 14 and $16 \mu \mathrm{m}$. The solid lines on the spectra represent fits of the spectra by thermal dust emission models. The blue lines correspond to a model of dust at an equilibrium temperature $T_{\mathrm{d}}$ varying from $145 \mathrm{~K}$ to $95 \mathrm{~K}$, as indicated on the plots. The red lines correspond to a model in which the dust is heated by collisions with the electrons of the hot plasma with density and temperature, $n_{\mathrm{e}}$ and $T_{\mathrm{e}}$, as indicated on each plot, and in which the grain size distribution is an $a^{-3.5}$ power law with a lower end cut-off at $a_{\text {min }}=30 \AA$ (see text). In all models, the optical constants for the grains were those of "astronomical" silicate (Draine \& Lee 1984).

at $54 \mathrm{~K}$ (Saken et al. 1992). The total mass of dust needed to account for the observations is about $10^{-4} M_{\odot}$.

As shown in Fig. 1, the spatial correlation between the IR emission and the optical emission is very good. Blair et al. (1991) reported that the Kepler optical emission is dominated by non-radiative Balmer hydrogen lines from filaments with a diffuse and knotty morphology. There is thus a general agreement that the optical emission mainly originates from shocked circumstellar material (Blair et al. 1991). The good spatial correlation between the IR emission and the optical emission leads us to claim that the origin of the thermal emission from Kepler is due to circumstellar dust. 


\subsection{Dust heating}

In order to further assess the link between the optical emission and the dust emission, we examined whether the dust could be collisionally heated by the hot electrons of the plasma in the regions emitting the optical lines; this plasma is thought to be generated by the secondary shocks driven into the dense clumps of circumstellar material by the SN blast wave. Observations of optical lines indicate that the plasma electron density, $n_{\mathrm{e}}$, ranges from $2000 \mathrm{~cm}^{-3}$ to more than $10000 \mathrm{~cm}^{-3}$ (Blair et al. 1991) and that the electron temperature, $T_{\mathrm{e}}$, is of the order of $10^{4} \mathrm{~K}$ (D'Odorico et al. 1986); note that these numbers are just guides, as the dust emission regions can be much broader than the optical line emission regions, which require specific conditions in terms of density and temperature, that are not required for dust heating. The dust parameters entering into the model are the dust composition, which is taken to be "astronomical silicates", and the dust size distribution, which is taken to be interstellar: an $a^{-3.5}$ power law (Mathis et al. 1977, hereafter MRN) with a lower end cut-off at a radius $a_{\text {min }}=30 \AA$ (Weinggartner \& Draine 1998; Kim et al. 1994; O’Donnell \& Mathis 1997) and an upper end cut-off at $0.1 \mu \mathrm{m}$. Figure 3 shows the result of a typical fit; for this fit, the parameters were $n_{\mathrm{e}}$ equal to $6000 \mathrm{~cm}^{-3}$ and $T_{\mathrm{e}}$ equal to $5 \times 10^{4}$. Good fits are also obtained by varying the density between 100 and $10000 \mathrm{~cm}^{-3}$, the temperature between $10^{4} \mathrm{~K}$ and $10^{6} \mathrm{~K}$, keeping the product $n_{\mathrm{e}} \cdot T_{\mathrm{e}}$ in the $2 \times 10^{8}-10^{9} \mathrm{~cm}^{-3} \mathrm{~K}$ range, and the cut-off grain size between $10 \AA$ and $90 \AA$.

In the previous fit, we have assumed that the physical conditions were the same throughout the remnant. In fact, the ISOCAM spectro-imaging observations indicate that this is not fully the case (see spectra of Fig. 2). The temperature corresponding to the fit of the ISOCAM spectra with "astronomical silicates" at thermal equilibrium ranges from $95 \mathrm{~K}$ for region $b$ of Fig. 2 to $145 \mathrm{~K}$ for region $c$ of Fig. 2. The two brightest regions (regions $a$ and $e$ ), which account for $60 \%$ of the total flux, have similar temperatures, so that the overall spectrum fit is only slightly changed when allowing for spatial variations of the dust temperature.

\section{Tycho}

The Tycho SNR is the remnant of a SN explosion which was observed by Tycho Brahe in 1572. The size of the remnant on the sky is $8^{\prime} \times 5^{\prime}$, which corresponds to $5.3 \mathrm{pc} \times 3.3 \mathrm{pc}$ at the distance of the remnant, known to be of $2.3 \mathrm{kpc}$ (Chevalier et al. 1980). The remnant has been extensively studied in X-rays (Hwang et al. 1998; Hwang \& Gotthelf 1997; Vancura et al. 1995; Fink et al. 1994), radio (Velázquez et al. 1998; Reynoso et al. 1997), and optical (Smith et al. 1991). The IR studies of Tycho are very scarce. The only results come from IRAS observations (Dwek et al. 1987; Arendt 1989; Saken et al. 1992; Braun 1997). Given the size of the remnant, it was spatially resolved with IRAS; an IRAS map of Tycho is presented in Schwarz et al. (1995).

\subsection{ISOCAM observations}

The ISOCAM observation of Tycho consists only of a full mapping of the remnant at a spatial resolution of $6^{\prime \prime}$ and with the LW8 filter. A raster map consisting of 5 by 5 frames of $3^{\prime}$ by $3^{\prime}$ each, with an overlap of $24^{\prime \prime}$ from one frame to the other, was performed on 12 January, 1997. A total field of $14.4^{\prime}$ by $14.4^{\prime}$, centered on the center of the remnant as deduced from X-ray observations (RA $00^{\mathrm{h}} 25^{\mathrm{m}} 21^{\mathrm{s}}$, Decl. $+64^{\circ} 08^{\prime} 06^{\prime \prime}$ 2000, Hwang \& Gotthelf 1997), was covered. A total time of 195 s, (93 elementary images with $2.1 \mathrm{~s}$ for each), was spent on each frame, so that the sensitivity reached on Tycho is almost the same as the sensitivity reached on Kepler. Nevertheless, given that the surface brightness of the object is fainter than that of Kepler, the signal to noise in the image is poorer for Tycho than for Kepler, so that CVF spectro-imaging follow-up observations would have required prohibitively long integration times. The data reduction follows exactly the same steps as for Kepler, and the result is shown in Fig. 4. The total flux from the remnant integrated over a $8^{\prime}$ diameter circle is $1.9 \mathrm{Jy}$ with an uncertainty of $\pm 0.8 \mathrm{Jy}$; (a non negligible contribution (0.56 Jy) from stars was subtracted from the total flux). The $12 \mu \mathrm{m}$ IRAS flux was very uncertain, ranging from $1.8 \pm 1.8 \mathrm{Jy}$ (Arendt 1989) to $3.07 \pm 0.3 \mathrm{Jy}$ (Braun 1987).

\subsection{Discussion}

Figure 4 shows a comparison between the ISOCAM image of Tycho and an $\mathrm{H}_{\alpha}$ image of the remnant. The relative astrometry between the two images was obtained by spatial correlation between the stars detected at both wavelengths. Most of the IR emission is spatially well correlated with the $\mathrm{H}_{\alpha}$ optical emission. In analogy to the case of Kepler, it can be claimed that the IR emission problably originates as thermal emission from circumstellar or interstellar material. The IR emission in the North-East of Fig. 4 has no optical counterpart. This IR emission, already detected by IRAS (especially at $100 \mu \mathrm{m}$ ), lies outside the SN blast wave and is probably not related to the SNR.

Figure 5 shows a fit of the ISOCAM and IRAS data, collected on Tycho, by a model of dust thermal emission. While a single dust component was able to account for the data on Kepler, Tycho's data cannot be similarly fitted. The dotted line of Fig. 5 represents the flux expected by thermal emission from dust collisionnally heated in the same conditions as for Kepler (see Sect. 2.5). The model can adequately fit the data at 11 and $25 \mu \mathrm{m}$, but does not predict enough flux at 60 and $100 \mu \mathrm{m}$. A second component of "Astronomical" silicates at a temperature of $55 \mathrm{~K}$ has to be introduced. A dust mass of $10^{-4} M_{\odot}$ was needed for the warm component and a dust mass of $4 \times 10^{-3} M_{\odot}$ 


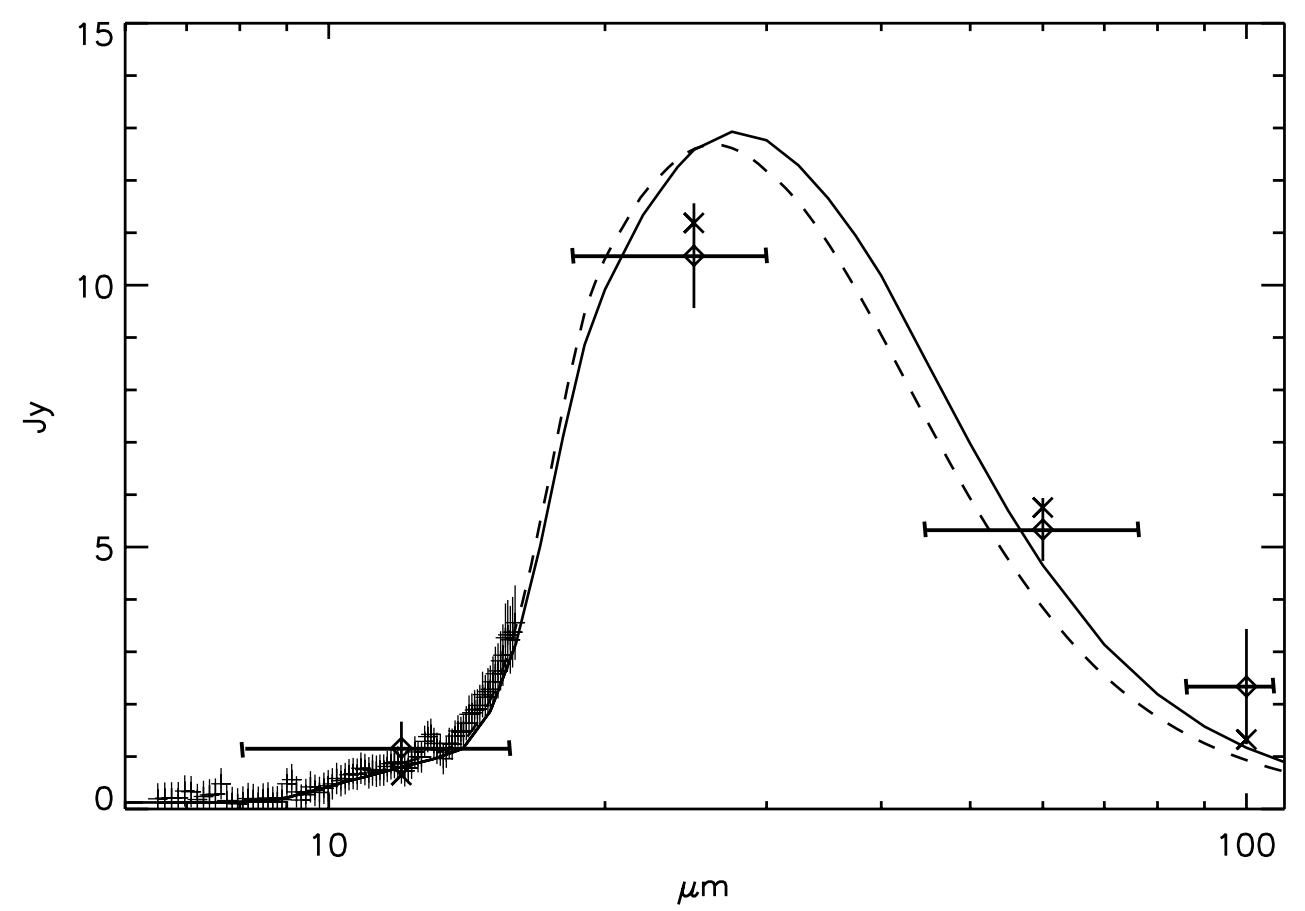

Fig. 3. Global spectrum of Kepler from ISOCAM CVF data (regions $a$ to $e$ of Fig. 2 spatially co-added), and IRAS data; the ISOCAM data are indicated with symbols + and IRAS data with squares. The full line corresponds to a fit of the data with a model of "astronomical silicate" collisionally heated by a hot plasma with electron density, $n_{\mathrm{e}}$, equal to $6000 \mathrm{~cm}^{-3}$ and electron temperature, $T_{\mathrm{e}}$, equal to $5 \times 10^{4} \mathrm{~K}$; the grain size distribution is as in Fig. 2. Crosses $(\times)$ are the fluxes in the IRAS bands as predicted by the model and should be compared with the squares; the squares are the IRAS data color corrected according to the full line fit; uncorrected IRAS values and error bars are from Arendt (1989). The dashed line corresponds to a fit with a model of thermal dust emission of "astronomical silicate" at an equilibrium temperature of 107.5 K. For readability, IRAS color corrected values with respect to this fit have not been overplotted (they are almost the same as the red ones).

was needed for the cold component. Note that the fit shown in Fig. 5 is not unique; for example, a two component dust model with one component at a temperature of $137 \mathrm{~K}$ and the other at $65 \mathrm{~K}$ is also able to fit the data. Additional observations are needed to assess the origin of the cold component.

\section{The Crab Nebula}

The Crab Nebula, the first entry in the Messier Catalog, is the remnant of a SN explosion which was recorded in 1054 by Chinese astronomers (Duyvendak 1942). The size of the remnant on the sky is about $4^{\prime} \times 4^{\prime}$, which corresponds to $2.4 \mathrm{pc} \times 2.4 \mathrm{pc}$ at the distance of the nebula, $2 \mathrm{kpc}$ (e.g. Trimble 1968). The nebula is one of the most studied objects in the Galaxy (see e.g. the review by Davidson \& Fesen 1985 and the references therein). It is the strongest, and historically the first identified, source of synchrotron radiation in our galaxy; the synchrotron radiation has been detected from radio to X-rays. The SN ejecta are seen in the form of filaments, which are photoionized by the synchrotron radiation (e.g. Henry \& MacAlpine 1982; Péquignot \& Dennefeld 1983). Few IR observations of the Crab Nebula have been made. IRAS observations have shown that the overall IR radiation was dominated by cold $(46 \mathrm{~K})$ dust thermal emission at long wavelengths (60 and $100 \mu \mathrm{m})$ and by synchrotron radiation at shorter wavelengths (12 and $25 \mu \mathrm{m})$. The presence of dust in the Crab Nebula has also been identified in absorption in optical images (Fesen \& Blair 1990; Hester et al. 1990; Blair 1997; Sankrit et al. 1998).

\subsection{ISOCAM observations}

The ISOCAM observations of the Crab Nebula presented here consists of CVF spectro-imaging observations of the central $3^{\prime} \times 3^{\prime}$ part of the nebula at a spatial resolution of $6^{\prime \prime} \times 6^{\prime \prime}$. The observations were performed on 22 February, 1998. The wavelength of the observations ranges from $6.5 \mu \mathrm{m}$ to $16 \mu \mathrm{m}$ and was covered by 122 different CVF positions, starting with the longest wavelength. A total integration time of $23 \mathrm{~s}$, (11 exposures of $2.1 \mathrm{~s}$ each), was spent for each CVF position. The CFV scan was first performed on a frame centered on the pulsar left of the SN explosion and then on a nearby field (RA $05^{\mathrm{h}} 34^{\mathrm{m}} 31.08^{\mathrm{s}}$, Decl. $\left.+21^{\circ} 40^{\prime} 53^{\prime \prime} 2000\right)$ in order to record the background level. Due to observing time limitations, the scan on the background only covered the $9.2-16 \mu \mathrm{m}$ wavelength range. The contribution of the zodiacal light in the wavelength range $6.5-9.2 \mu \mathrm{m}$ was determined by scaling specific zodiacal observations (Reach et al. 1996) to the $9.2-16 \mu \mathrm{m}$ background spectrum near the Crab Nebula.

When using the CFV, ghost effects and detector transient effects are sources of uncertainty. The ghost problem 


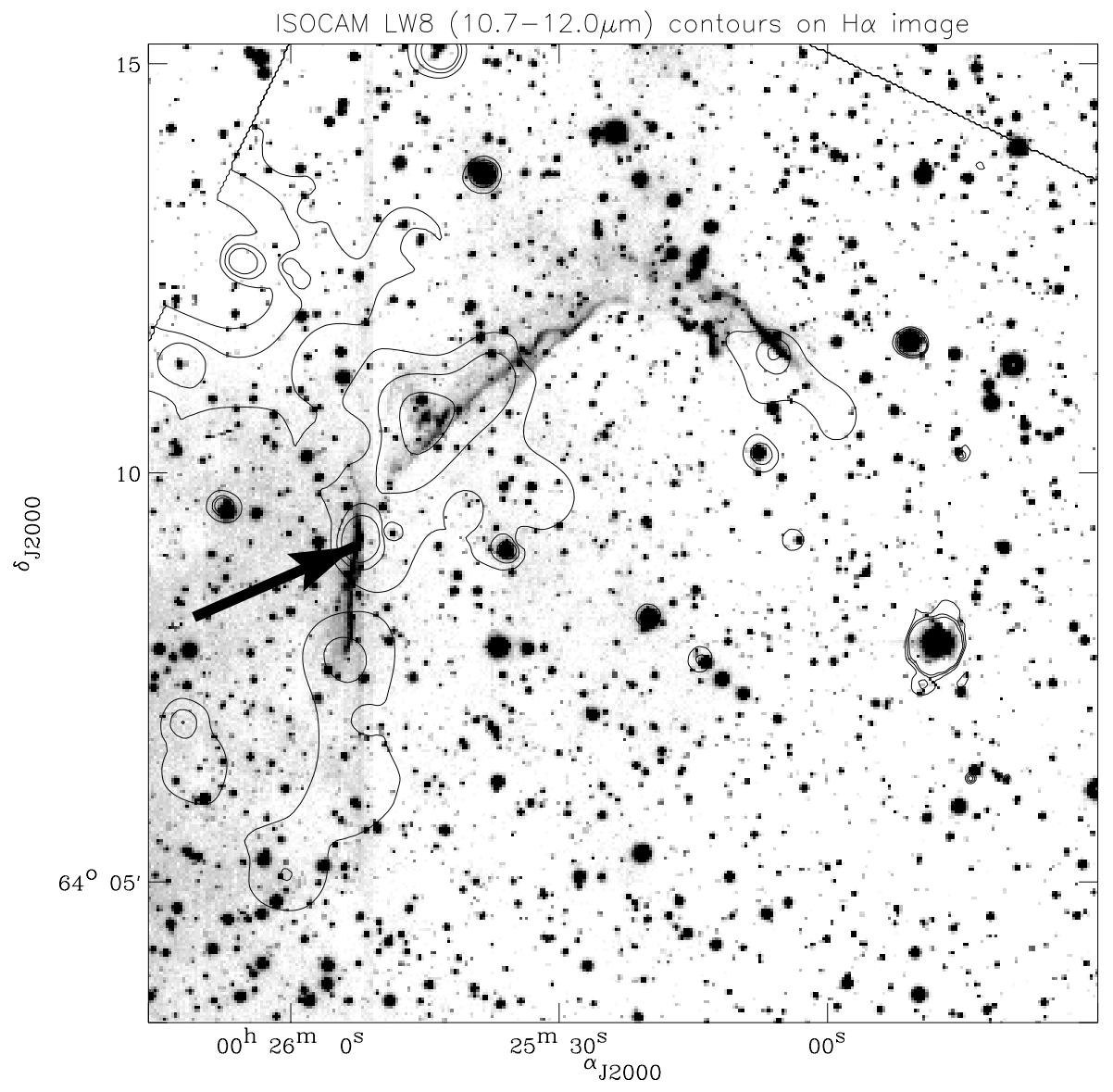

Fig. 4. Contours of the ISOCAM image of the Tycho SNR as observed with the LW8 filter. The highest contour level, shown by the arrow, represents a flux level of $2.8 \mathrm{mJy}$ per ISOCAM pixel $\left(6^{\prime \prime} \times 6^{\prime \prime}\right)$; the contour level is reduced by $0.2 \mathrm{mJy}$ at each subsequent contour. Underlying the IR contours is an $\mathrm{H}_{\alpha}$ image of the remnant, courtesy of Ghavamian and Blair; the image was taken with the $4 \mathrm{~m}$ telescope of the KPNO in 1991.

is alleviated when using the ISOCAM broad-band filters; the transient effects can be limited by long integration times in a given configuration. Thus, a second set of data was obtained; this second set consists of an integration time of $105 \mathrm{~s}$ with the LW1 filter, (centered at $4.5 \mu \mathrm{m}$ and with a $F W H M$ of $1 \mu \mathrm{m}$ ), and of $73.5 \mathrm{~s}$ with the LW8 filter. The observations were performed on the same date, with the pointing and the same pixel field of view as the CVF observations. The "beam switching" Astronomical Observing Template (AOT number 3) was used, so that a background image was taken as part of the observation.

\subsection{Results}

The data reduction was the same as for Kepler or Tycho. Results of the CVF observations are shown in Fig. 6. The spectra feature both ionic line emission and continuum emission. In the IRAS band $(8-15 \mu \mathrm{m})$, the continuum emission contributes to $77 \%$ of the total flux. Ionic lines of [Ne II], [Ne III], [Ne V] at respectively 12.8, 15.5 and $14.3 \mu \mathrm{m}$, of [Ar II], [Ar III] at $7.0 \mu \mathrm{m}$ and $9.0 \mu \mathrm{m}$ and of [S IV] at $10.5 \mu \mathrm{m}$ can be easily identified. A fainter line, tentatively attributed to $[\mathrm{NiII}]$, is present at $6.7 \mu \mathrm{m}$. A map of the [NeIII] emission line is shown in Fig. 6; the map shows a filamentary structure, similar to the structure seen in ionic lines in the optical (see e.g. Hester et al. 1990); these filaments are known to be composed predominately of supernova ejecta. A map of the continuum emission is also shown in Fig. 6; the spatial structure of this map is completely different to that of [NeIII] line emission and is similar to the synchrotron nebula seen at other wavelengths (radio, optical, X-rays). No spectral feature from thermal dust emission is detected in any of the ISOCAM spectra, even at places where dust was seen in absorption in the optical.

\subsection{Synchrotron spectral index}

There is general agreement that the spatially integrated synchrotron spectrum of the Crab Nebula has a spectral index $\alpha$ varying from $-0.27 \pm 0.04$ in the radio (Baars \& Hartsuijker 1972; Mezger et al. 1986) to -0.8 in the optical (e.g. Véron-cetty \& Woltjer 1993), (the spectral index, $\alpha$, is defined as $F_{\nu} \propto \nu^{\alpha}$, where $F_{\nu}$ is the flux at frequency $\nu$ ). The break between the two indexes was claimed to be in the mid-IR at $1.4 \times 10^{13} \mathrm{~Hz}(21 \mu \mathrm{m})$ (Strom \& Greidanus 1992). It is difficult to deduce a precise spectral index from the overall spectrum of Fig. 6, because of the uncertainties in the CFV observations. To calculate the spectral index, we have used the observations performed with the LW1 and the LW8 filters; a value of $-0.50 \pm 0.10$ was found. This value is intermediate between the radio and optical values, indicating that we are probing the wavelength region where the break in the spectral index occurs.

There is a potential caveat in the previous result: the IR spectral index was deduced from the observations of the 


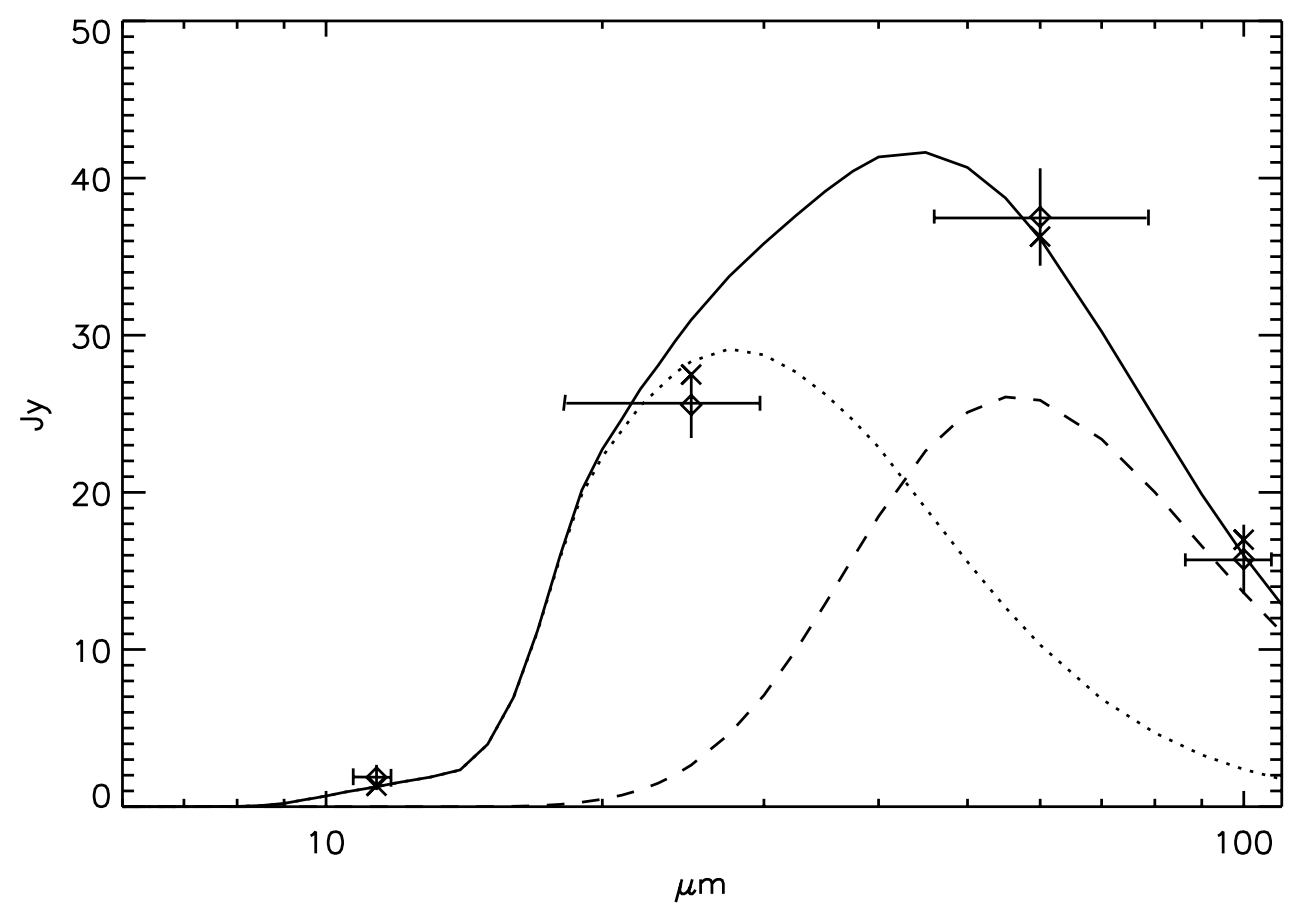

Fig. 5. Fit (full line) of the IR data, (ISOCAM data at $11.3 \mu \mathrm{m}$ and the 3 IRAS data at $25 \mu \mathrm{m}, 60 \mu \mathrm{m}$ and $100 \mu \mathrm{m}$ ), on Tycho with dust thermal emission. The dotted line represents the thermal emission of dust collisionally heated as in Kepler (see caption of Fig. 3); a second colder $(55 \mathrm{~K})$ component (dashed line) has to be added in order to reproduce the data. Crosses $(\times$ ) are the fluxes predicted by the model in the LW8 ISOCAM filter band or in the IRAS bands and should be compared with the squares; the squares are the IRAS data, color corrected according to the full line curve.

central $3^{\prime} \times 3^{\prime}$ part of the Crab, while the spectral indexes to be compared were calculated over the whole spatial extent of the nebula. While no (or little) spatial variation in the spectral index was found in the radio (Bietenholz et al. 1997), spatial variation was found in the optical (Véroncetty \& Woltjer 1993). Spatial variation in the spectral index is also observed in the ISOCAM data, as can be seen in Fig. 7; the spectral index varies from -0.3 in the inner part to -0.65 at the edge of the observed field; this spatial variation should be compared to -0.6 to -0.9 in the case of visible observations (Véron-cetty \& Woltjer 1993). Thus, we can conclude that the spectral index in the mid-IR is definitely intermediate between the optical and the radio.

The map of the spectral index is spatially well correlated with the synchrotron nebula itself; no trace of the filaments is visible. Velusamy et al. (1992) suggested that the magnetic field increases in the filaments, and therefore that the energy loss of electrons within filaments are more significant than in the nebular region. Nevertheless, they themselves reported, in agreement with Swinbank (1980), that for the filaments in the inner parts of the remnant $\left(r<100^{\prime \prime}\right)$, no evidence for a variation in the spectral index is shown. Additionally, from our observations, we conclude that there is no evidence for the spectral index spatial variations to be correlated with the filaments.

There is not yet a comprehensive model accounting for all the Crab synchrotron observations. Simple models (Pacini \& Salvati 1973; Amato et al. 2000), with as the main ingredient an expanding bubble into which relativistic electrons and magnetic fields are constantly injected by the pulsar, are able to account for the overall synchrotron spectrum and the decline in time of the luminosity, but fail to reproduce the spatial variation of the flux observed in the optical. More sophisticated models (Kennel \& Coroniti 1984; Atoyan \& Aharonian 1996), taking into account the propagation of electrons, are able to account for both the overall spectrum and the spatial variation of the flux in the optical, but at the price of introducing ad-hoc two populations of relavistic electrons, one at the origin of the radio/far-IR synchrotron radiation and the other at the origin of the synchrotron radiation at optical and shorter wavelengths. Our observations are in the crucial region where both populations contribute, so that detailed comparison of the model with observation is needed; such a comparison is beyond the scope of this paper.

\section{Conclusion and discussion}

The analysis of the mid-IR ISOCAM observations of Kepler, Tycho and the Crab, combined with $\mathrm{H}_{\alpha}$ and IRAS data, has led to the following conclusions:

- (1) The mid-IR emission from Kepler and Tycho is spatially well correlated with the $\mathrm{H}_{\alpha}$ optical emission;

- (2) Tycho and Kepler show no evidence of dust newly formed from SN ejecta;

- (3) The IR spectrum of Kepler is well modeled by thermal emission from $10^{-4}-2 \times 10^{-4} M_{\odot}$ of "astronomical silicates" (Draine \& Lee 1984) at a temperature of $107 \mathrm{~K}$; 

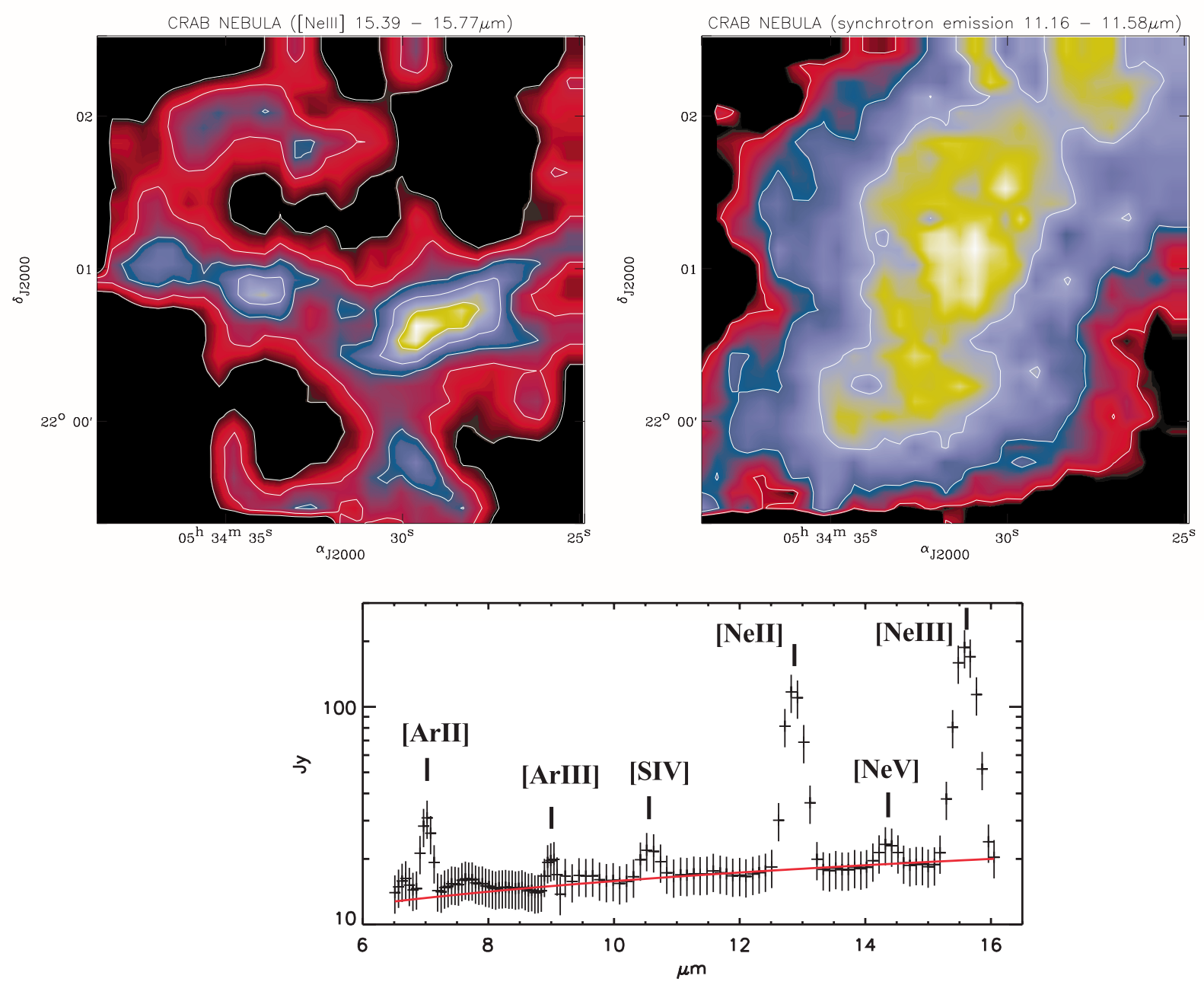

Fig. 6. Left image: ISOCAM image of the Crab Nebula filaments in the Neon III line at $15.5 \mu$ m (the image was obtained by integrating fluxes between 15.39 and $15.77 \mu \mathrm{m}$ and by subtracting the underlying continuum, shown as the full line). Right image: ISOCAM image of the synchrotron emission at 11.16 and $11.58 \mu \mathrm{m}$. Super-imposed on the images are the contour plots associated with the image; at each contour the flux level is divided by a factor 1.5. Bottom: overall spectrum of the Crab nebula (obtained by co-adding the spectra of all individual pixels in the image, except those on the edges of the image); the spectrum features ionic forbidden lines superimposed onto a flat continuum synchrotron emission. The full line represents the spectrum of the synchrotron radiation with a spectral index of -0.5 , deduced from observations with the filters LW1 and LW8 (see text Sect. 4.2).

- (4) Dust collisional heating is a natural mechanism to explain the dust temperature;

- (5) In the case of Tycho, in addition to a warm component similar to the component in Kepler, a cold $(55 \mathrm{~K})$ component of $4 \times 10^{-3} M_{\odot}$ has to be introduced. This component is probably not related to the SNR itself;

- (6) No dust can be seen in the Crab Nebula at mid-IR wavelengths;

- (7) The mid-IR spectral index of the synchrotron radiation in the Crab Nebula varies from -0.3 to -0.65 , according to the distance to the pulsar.

The most striking result of the observations of Kepler and Tycho is the spatial correlation between the optical line emission and the mid-IR radiation in Tycho and Kepler; such a correlation was also found in the case of Cas A
(Lagage et al. 1996). The correlation holds mainly because the temperature and electron density of the gas emitting optical lines is such that the heating of the dust by electronic collisions is efficient; the dust can reach a temperature high enough $(100 \mathrm{~K})$ that a significant fraction of the dust thermal emission is emitted in the mid-IR and is detectable by ISOCAM. The mass of dust found in Kepler $\left(10^{-4}-2 \times 10^{-4} M_{\odot}\right)$ is about $1 \%$ of the mass of ionized hydrogen (D'Odorico et al. 1986). Given that the gas seen in the optical does not originate from SN ejecta, the previous arguments lead us to conclude that we do not observe in Kepler dust made from SN material. Fitting both the ISOCAM mid-IR data and the IRAS data at longer wavelengths with the same dust component leaves little room for an extra dust component made of supernova material. The only remaining possibility would 


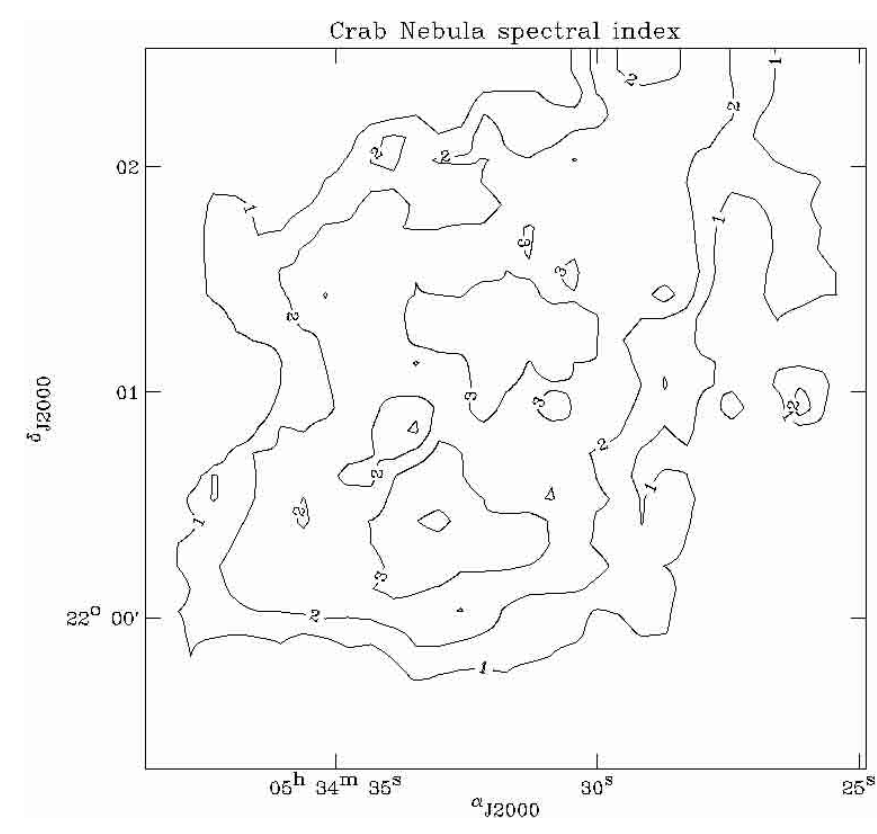

Fig. 7. The plain lines are the iso-levels of spectral index of the synchrotron radiation from the Crab Nebula; the labels 1, 2,3 correspond to a spectral index of respectively $-0.3,-0.45$, -0.65 .

be that SN dust is in the form of cold unshocked knots. The minimum temperature of such knots, only heated by the radiation field, would be $17 \mathrm{~K}$ (Dwek \& Werner 1981). Given the IRAS constraint at $100 \mu \mathrm{m}$, only $0.1 M_{\odot}$ of such a cold dust could be present in Kepler. It is difficult to compare this upper limit with expected values. Indeed, the mass of the Kepler progenitor is unknown; even the type of SN is under debate. When Badde (1943, 1945) claimed that the historical light curve favours type Ia SNe for Kepler, Doggett and Branch (1985) showed that the curves were also consistent with type II SNe. More recently, the SN type was discussed by Decourchelle \& Ballet (1994) who concluded that Kepler is probably not a type Ia SN. Nevertheless, the interpretation of both $\mathrm{X}$-ray and optical radiations in Kepler is problematic. It is generally admitted that the optical nebulosities are not associated with supernova ejecta, but the very good spatial correlation between X-ray emission and optical emission is striking. Some authors (Decourchelle \& Ballet 1994; Decourchelle \& Petre 1999) try to obtain the best consistence only regarding the X-ray data, but their best model (a type II supernova without wind in the presupernova stage) is inconsistent with the origin and the location of the optical nebulosities. Some other authors (Borkowski et al. 1994; Borkowski et al. 1992) prefer a more consistent model regarding the origin of the optical nebulosities, and generally more consistent with the morphology of the remnant at all wavelengths, but it poorly explains the Xray data, particularly the X-ray spectra. If Kepler were a type II SN and if we assume that the dust production efficiency is $100 \%$, then the upper limit of $0.1 M_{\odot}$ suggests an upper limit of $15 M_{\odot}$ for the SN precursor (Woosley \& Weaver 1995). If as discussed in Douvion et al. (1999), the mixing is incomplete, then the dust efficiency is reduced and the limit of $0.1 M_{\odot}$ is still compatible with a SN precursor mass up to $25 M_{\odot}$. If Kepler has a progenitor of lower mass, so that the SN was of type IA, then the limit of $0.1 M_{\odot}$ is still compatible with the possibility that the dust was produced with high efficiency after the SN explosion (Iwamoto et al. 1999).

The type of the Tycho SN is also under debate; in addition, because of the presence of the cold dust component which is probably not related to the SNR, it is not possible to derive a useful upper limit regarding a possible cold dust component made of SN ejecta. To proceed, we await a sub-millimeter facility providing good spatial resolution, such as the FIRST facility to be launched in 2007 (Genzel 1997).

Acknowledgements. We thanks A. Decourchelle and J. Ballet for helpfull discussions on physical conditions in Kepler. We thank O. Hainaut who performed the service mode SOFI observations of Kepler. We are also grateful to Ghavamian and Blair who provided us with the $\mathrm{H}_{\alpha}$ image of Tycho.

\section{References}

Amato, E., Salvati, M., Bandiera, R., Pacini, F., \& Woltjer, L. 2000, preprint [astro-ph/9911163]

Arendt, R. G. 1989, ApJS, 70, 181

Arendt, R. G., Dwek, E., \& Moseley, S. H. 1999, ApJ, 521, 234

Atoyan, A. M., \& Aharonian, F. A. 1996, A\&AS, 120, 453

Baade, W. 1943, ApJ, 97, 119

Baade, W. 1945, ApJ, 102, 309

Baars, J. W. M., \& Hartsuijker, A. P. 1972, A\&A, 17, 172

Bietenholz, M. F., Kassim, N., Frail, D. A., et al. 1997, ApJ, 490, 291

Blair, W. P., Long, K. S., \& Vancura, O. 1991, ApJ, 366, 484,

Blair, W. P., et al. 1997, ApJS, 109, 473

Borkowski, K. J., Blondin, J. M., \& Sarazin, C. L. 1992, ApJ, 400, 222

Borkowski, K. J., Sarazin, C. L., \& Blondin, J. M. 1994, ApJ, 429, 710

Bouchet, P., Danziger, I. J., \& Lucy, L. B. 1991, AJ, 102, 1135

Braun, R. 1987, A\&A, 171, 233

Cernushi, F., Marsicano, F. \& Codina, S. 1967, Ann. d'Ap., 30, 1039

Cesarsky, C. J., Abergel, A., et al. 1996, A\&A, 315, L32

Chevalier, R. A., Kirshner, R. P., \& Raymond, J. C. 1980, ApJ, 235,186 ,

Moon and Planets, 19, 109 (1978)

Colgan, S. W. J., Haas, M. R., Erickson, E. F., Lord Steven, D., \& Hollenbach, D. J. 1994, ApJ, 427, 874

Coulais, A., Abergel, A. 1999, ESA Proceedings SP-427, The Universe as seen by ISO, ed. P. Cox, \& M. F. Kessler, 61

Davidson, K., Fesen, \& R. A. 1985, ARA\&A, 23, 119

Decourchelle, A., \& Ballet, J. 1994, ARA\&A, 287, 206

Decourchelle, A., \& Petre, R. 1999, Astron. Nachr., 3, 152

Dickel, J. R., Sault, R., Arendt, R. G., Matsui, Y., \& Korista, K. T. 1988, ApJ, 33, 254

Doggett, J. B., \& Branch, D. 1945, AJ, 90, 2303

D'Odorico, S., Bandiera, R., Danziger, J., \& Focardi, P. 1986, AJ, 91, 1382

Douvion, T., Lagage, P. O., \& Cesarsky, C. J. 1999, AJ, in press 
Douvion, T., Lagage, P. O., Ballet, J., et al. 1999, ESA Proceedings, SP-427, The Universe as seen by ISO, ed. P. Cox, \& M. F. Kessler, 301

Draine, B. T., \& Lee, H. M. 1984, ApJ, 2885, 89

Draine, B. T., \& Salpeter, E. E. 1979, ApJ, 231, 77

Duyvendak, J. J. L. 1942, PASP, 54, 91

Dwek, E., \& Werner, M. W. 1981, ApJ, 248, 138

Dwek, E. 1986, ApJ, 302, 363

Dwek, E., Petre, R., Szymkowiak, A., \& Rice, W. L. 1987, ApJ, 320, L27

Dwek, E. 1998, ApJ, 501, 643

Fesen, R. A., \& Blair, W. P. 1990, ApJ, 351, L45

Fink, H. H., Asaoka, I., Brinkmann, W., Kawai, N., \& Koyama, K. 1994, ApJ, 283, 635

Genzel, R. 1997, ASP Conf. Ser. 124, Diffuse Infrared Radiation and the IRTS, ed. H. Okuda, T. Matsumoto, \& T. Rollig, 465

Henry, R. B. C., \& MacAlpine, G. M. 1982, ApJ, 258, 11

Hester, J. J., Graham, J. R., Beichman, C. A., \& Gautier III, T. N. 1990, ApJ, 357, 539

Hoyle, F., \& Wickramasinghe, N. C. 1970, Nature, 226, 62

Hughes, J. P. 1999, ApJ, in press

Hwang, U., \& Gotthelf, E. V. 1997, ApJ, 475, 665

Hwang, U., Hughes, J. P., \& Petre, R. 1998, ApJ, 497, 833

Iwamoto, K., Brachwitz, F., Nomoto, K., et al. 1999, ApJS, 125,439

Kennel, C. F., \& Coroniti, F. V. 1984, ApJ, 283, 710

Kessler, M. F., et al. 1996, A\&A, 315, L27

Kim, S.-H., Martin, P. G., \& Hendry, P. D. 1994, ApJ, 422, 164

Kozasa, T., Hasegawa, H., \& Nomoto, K. 1991, A\&A, 249, 474

Kozasa, T., Nomoto, K., \& Shigeyama, T. 1997, ASP Conf. Ser., Dust Formation in SN 1987 A, The Fifth CTIO/LCO Workshop, ed. N. B. Suntzeff, \& M. M. Phillips

Lagage, P. O., Claret, A., Ballet, J., et al. 1996, A\&A, 315, L273

Lucy, L. B., Danziger, I. J., Gouiffes, C., \& Bouchet, P. 1989, IAU Colloq. 120, ed. G. Tenorio-Tagle, M. Moles, \& J. Melnick (Berlin: Springer-Verlag), 164

Mathis, J. S., Rumpl, W., \& Nordsieck, K. H. 1977, ApJ, 217, 425

Mezger, P. G., Tuffs, R. J., Chini, R., Kreysa, E., \& Gemünd, H. P. 1986, A\&A, 167, 145
Moseley, S. H., Dwek, E., Glaccum, W., Graham, J. R., \& Loewenstein, R. F. 1989, Nature, 340, 697

O'Donnell, J. E., \& Mathis, J. S. 1997, ApJ, 479, 806

Ostriker, J. P., \& Silk, J. 1973, ApJ, 184, L113

Pancini, F., \& Salvati, M. 1973, ApJ, 186, 249

Péquignot, D., \& Dennefeld, M. 1983, A\&A, 120, 249

Reach, W. T., Abergel, A., Boulanger, F., et al. 1996, A\&A, 315, L381

Reynoso, E. M., Moffett, D. A., Goss, W. M., et al. 1997, ApJ, 491, 816

Rothenflug, R., Magne, B., Chièze, J. P., \& Ballet, J. 1994, A\&A, 291, 271

Saken, J. M., Fesen, R. A., \& Shull, J. M. 1992, ApJS, 81, 715

Schaefer, B. E. 1996, ApJ, 459, 438

Schwarz, U. J., Goss, W. M., Kalberla, P. M., \& Benaglia, P. 1995, A\&A, 299, 193

Silk, J., \& Burke, J. R. 1974, ApJ, 190, 11

Sankrit, R., et al. 1998, ApJ, 504, 344

Smith, A., Peacock, A., Arnaud, M., et al. 1989, ApJ, 347, 925

Smith, R. C., Kirshner, R. P., Blair, W. P., \& Winkler, P. F. 1991, ApJ, 375, 652

Starck, J. L., Murtagh, F., \& Bijaoui, A. 1995, ASP Conf. Ser. 77, Astronomical Data Analysis Software and Systems IV, ed. R. A. Shaw, H. E. Payne, \& J. J. E. Hayes, 279

Strom, R. G., \& Greidanus, H. 1992, Nature, 358, 654

Suntzeff, N. B., Phillips, M. M., Depoy, D. L., Elias, J. H., \& Walker, A. R. 1991, AJ, 102, 1118

Swinbank, E. 1980, MNRAS, 193, 451

Trimble, V. 1968, AJ, 73, 535

Trimble, V. 1983, Rev. Mod. Phys., 55, 511

Vancura, O., Gorenstein, P., \& Hughes, J. P. 1995, ApJ, 441, 680

Velázquez, P. F., Gómez, D. O., Duhner, G. M., Giménez de Castro, G., \& Costa, A. 1998, A\&A, 334, 1060

Velusamy, T., Roshi, D., \& Venugopal, V. R. 1992, MNRAS, 255,210

Véron-cetty, M. P., \& Woltjer, W. 1993, A\&A, 270, 370

Weingartner, J. C., \& Draine, B. T. 1999, ApJ, 517, 292

Wooden, D. H., Rank, D. M., Bregman, J. D., et al. 1993, ApJS, 88, 477

Woosley, S. E., \& Weaver, T. A. 1995, ApJS, 101, 181

Zinner, E. 1996, ASP Conf. Ser., 99, Cosmic Abundances, ed. S. S. Holt, \& G. Sonneborn, 147 